\title{
Mittel-Dosis-UVA-1- und lokale PUVA-Therapie beim dyshidrosiformen Handekzem - eine prospektive randomisierte Studie
}

\author{
Medium-Dose-UVA-1 Irradiation - and Topical PUVA - Therapy in Chronic Dyshidrotic Hand Dermatitis - \\ A Prospective Randomized Study
}

Autoren

Institute

\section{S. Adams ${ }^{1}$, C. Bayerl}

Hautklinik, Klinikum Dortmund gGmbH

Klinik für Dermatologie und Allergologie, HSK Wilhelm-Fresenius-Klinik, Städtisches Klinikum Wiesbaden

\section{Bibliografie}

Dol $10.1055 / \mathrm{s}-2007-966365$

Akt Dermatol 2007; 33:

142-145 @ Georg Thieme

Verlag KG Stuttgart · New York ISSN 0340-2541

Korrespondenzadresse

Prof. Dr. med. Christiane Bayerl

Klinik für Dermatologie und Allergologie

Städtische Kliniken Wiesbaden Lehrkrankenhaus der Universität Mainz

HSK, Wilhelm-Fresenius-Klinik $\mathrm{GmbH}$

Aukammallee 39

65191 Wiesbaden christiane.bayerl@hsk-

wiesbaden.de

\section{Zusammenfassung \\ $\nabla$}

Diese Studie zielte darauf ab, zwei unterschiedliche Behandlungsregimes für das dyshidrosiforme Handekzem zu vergleichen, die mitteldosierte UVA-1 Bestrahlung und die lokale Creme-PUVATherapie (topisches Psoralen und UVA). 15 Patienten wurden in einer prospektiven, randomisierten (rechte vs. linke Hand) Studie über fünf Wochen $3 \times$ pro Woche bestrahlt. Die mit der 0,001\%igen 8-Methoxypsoralen-Creme (Creme-PUVA) behandelte Hand wurde mit wöchentlich ansteigenden UVA-Dosen bestrahlt (kumulative UVA-Dosis $17,4 \mathrm{~J} / \mathrm{cm}^{2}$ ). Bei der mit Mittel-Dosis-UVA-1 behandelten Hand wurde die Dosis von $40 \mathrm{~J} / \mathrm{cm}^{2}$ beibehalten (kumulative UVA-1-Dosis $600 \mathrm{~J} / \mathrm{cm}^{2}$ ). Um die therapeutischen Effekte zu vergleichen, wurde ein Schweregradscore, der sog. DASI (Dyshidrotic Eczema Area and Severity Index) verwandt. Von 15 eingeschlossenen Patienten gingen

\section{Einleitung}

$\nabla$

Die Behandlung des dyshidrosiformen Handekzems stellt den Dermatologen im klinischen Alltag häufig vor Probleme. Nicht nur aufgrund der ausgesprochenen Rezidivfreudigkeit und der Neigung zur Chronizität, sondern auch aufgrund der Polyätiologie des Krankheitsbildes gestaltet sich eine kausale Therapie oft sehr schwierig, die Ursachensuche ist vielfach kostspielig. Therapeutisch stehen neben allgemeinen Hautschutzmaßnahmen v.a. symptomatisch wirksame Lokalbehandlungen mit Glukokortikosteroiden im Vordergrund. Das Behandlungsspektrum umfasst weiterhin eine Vielzahl lichttherapeutischer Verfahren, die Pinsel-PUVA- [5], Bade-PUVA- [2,18], Creme-PUVA- $[2,14]$, UVB- [1] und die UVA-1Therapie $[14,15,19]$. Die Pinsel-PUVA-Therapie hat das Risiko unerwünschter phototoxischer Reaktionen beim Abtropfen der Lösung. Die Vorbe-
11 in die Endauswertung ein $(1 \times$ non Compliance, $1 \times$ bakterielle Sekundärinfektion, $1 \times$ Exazerbation, $1 \times$ nicht studienbedingte Erkrankung). Nach insgesamt 15 Behandlungen führte sowohl die Creme-PUVA-Therapie $(p=0,0498 ; n=11)$, als auch die UVA-1-Therapie ( $p=0,0039 ; n=11$ ) verglichen mit den erhobenen Befunden vor Behandlungsbeginn zu einer signifikanten Besserung des Handekzem-Schweregrades (Wilcoxon-Test für verbundene Stichproben). Bei einem p-Wert von $p=0,307$ konnte kein Unterschied zwischen beiden Therapieformen bzgl. ihrer Wirksamkeit nachgewiesen werden (U-Test von Mann und Whitney). Jede der beiden Therapieformen für sich genommen war wirksam (Reduktion des DASI um mehr als die Hälfte) und nebenwirkungsarm. Der therapeutische Effekt war mit geringeren kumulativen Dosen zu erzielen als bislang publiziert.

reitung der Bade-PUVA-Behandlung ist bedingt durch die benötigten Ressourcen aufwendig. Die Bestrahlung mit UVB wird aufgrund einer fraglichen Karzinogenese zurückhaltend gesehen. Daher wurden zwei praktikable, einfach zu handhabende Bestrahlungmöglichkeiten, die CremePUVA- und die UVA-1-Therapie in dieser prospektiven, randomisierten Halbseitenstudie hinsichtlich ihrer Wirksamkeit und ihres Nebenwirkungsspektrums untersucht.

\section{Patienten und Methodik $\nabla$}

\section{Patientenauswahl}

Das Mindestalter der Patienten wurde auf 18 Jahre festgelegt. In die Studie einbezogen wurden Patienten, deren dyshidrosiformes Handekzem entweder chronisch rezidivierend auftrat, therapieresistent war oder aber länger als einen Monat bestand. 
Tab. 1 Bestrahlungsdosis in den beiden Therapiegruppen, je 3 Bestrahlungen pro Woche

\begin{tabular}{|c|c|c|c|c|c|c|c|c|c|c|c|c|c|c|c|}
\hline Behandlung & 1 & 2 & 3 & 4 & 5 & 6 & 7 & 8 & 9 & 10 & 11 & 12 & 13 & 14 & 15 \\
\hline $\begin{array}{l}\text { Dosis J/cm² } \\
\text { Creme-PUVA }\end{array}$ & 0,3 & 0,3 & 0,3 & 0,5 & 0,5 & 0,5 & 1,0 & 1,0 & 1,0 & 1,5 & 1,5 & 1,5 & 2,5 & 2,5 & 2,5 \\
\hline $\begin{array}{l}\text { Dosis } \mathrm{J} / \mathrm{cm}^{2} \\
\text { UVA-1 }\end{array}$ & 40 & 40 & 40 & 40 & 40 & 40 & 40 & 40 & 40 & 40 & 40 & 40 & 40 & 40 & 40 \\
\hline
\end{tabular}

Aufgrund der klinischen Beurteilung bzw. einer mykologischen Kultur wurden Patienten mit Psoriasis pustulosa, hyperkeratotisch rhagadiformen oder lichenifizierten Handekzemen sowie Patienten mit Tinea manuum ausgeschlossen. Als weitere Ausschlusskriterien galten: Schwangere oder stillende Patientinnen; einwilligungsunfähige oder unkooperative Patienten; Patienten, die eine PUVA- oder eine andere Lichttherapie während der letzten vier Wochen bzw. eine topische Glukokortikosteroidtherapie während der letzten Woche vor Studienbeginn erhalten hatten; Patienten, die bereits mehr als 200 PUVA-Behandlungen erhalten hatten; Patienten mit Medikamenten- oder Alkoholabusus; Patienten unter immunsuppressiver Therapie; Patienten mit anamnestisch bekannten Hauttumoren, UV-sensitiven Photodermatosen, Arsentherapie oder ionisierender Strahlentherapie in der Vorgeschichte; Patienten mit Begleittherapien außer lokal rückfettenden Maßnahmen. Als relatives Ausschlusskriterium galt die Einnahme lichtsensibilisierender Medikamente. Ein positives Votum der Ethikkommission II der Fakultät für Klinische Medizin Mannheim lag vor.

In die Studie eingeschlossen wurden insgesamt fünfzehn Patienten mit der Diagnose „dyshidrosiformes Handekzem“. Die Patienten wurden in der Lichtabteilung der Klinik für Dermatologie, Venerologie und Allergologie des Universitätsklinikums Mannheim behandelt.

\section{Auswahl des Bestrahlungsschemas}

Vor Behandlungsbeginn wurden die Patientenhandflächen (rechts versus links) randomisiert (Randombriefe wurden nach Würfeln von einer von der Studie unabhängigen Person erstellt). Über einen fünfwöchigen Therapiezeitraum unterzogen sich die Patienten insgesamt fünfzehn Behandlungen mit wöchentlichen Kontrollen. Bei der Wahl der 8-MOP-Konzentration und der Einwirkungszeit der Creme vor Bestrahlung folgten wir den Empfehlungen von Grundmann-Kollmann et al. [3]. Die mit 0,001\%iger 8-MOP-Creme (Rezeptur: 8-MOP 0,001; 1,2-Propylenglykol 9,4; Ethanol 96\% 6,0; Dest. Wasser 37,6; Basiscreme DAC 47,0) behandelte Hand wurde nach halbstündiger Einwirkungszeit mit wöchentlich ansteigenden UVA-Dosen bestrahlt ( Tab. 1). Die kumulative UVA-Dosis betrug 17,4 J/ $\mathrm{cm}^{2}$. Für die Behandlung wurde das Teilbestrahlungsgerät UV 200 AL der Firma Waldmann Lichttechnik eingesetzt (UVA-Strahler vom Typ Waldmann F8 T5/PUVA). Bei der mit Mittel-Dosis-UVA-1 behandelten Hand wurde die Dosis von $40 \mathrm{~J} / \mathrm{cm}^{2}$ über den gesamten Therapiezeitraum beibehalten, wobei sich die Wahl der Dosishöhe an den Veröffentlichungen von Polderman et al. und Schmidt et al. orientierte $[15,19]$. Die kumulative UVA-1-Dosis betrug $600 \mathrm{~J} / \mathrm{cm}^{2}$. Verwendet wurde die UVA-1-Lichttherapieliege medisun 24000 der Firma Schulze \& Böhm GmbH. Eine Verblindung der Studie erfolgte nicht. Vor Behandlungsbeginn und nach Behandlungsende wurde eine Fotodokumentation durchgeführt. Um die therapeutischen Effekte zu vergleichen, wurde ein Schweregradscore, der sog. DASI (Dyshidrotic Eczema Area and Severity Index) [24] verwandt, der für jede Hand getrennt

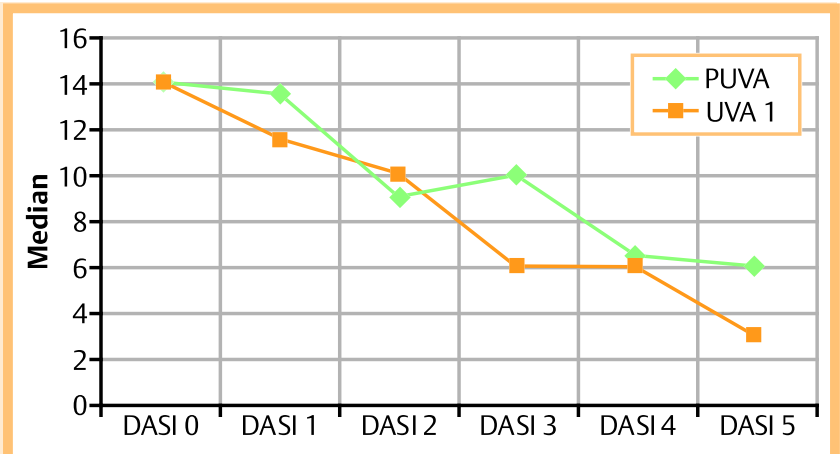

Abb. 1 Vergleich der Mittelwerte der Creme - PUVA - gegen die UVA- 1 - Therapie. DASI 0 = Studienstart, DASI 1 = nach der ersten Bestrahlungswoche, usw. DASI 5 = bei Studienende in der fünften Bestrahlungswoche.

ermittelt wurde. Dieser berücksichtigt die Kriterien Bläschen/ $\mathrm{cm}^{2}(V)$, Erythem $(E)$, Schuppung $(S)$ sowie das subjektive Kriterium Juckreiz $(I)$. Jeder dieser Unterpunkte wurde anhand einer Skala mit 0-3 Punkten $(\mathbf{P})$ bewertet $(0=$ fehlend, $1=$ mild/leicht, 2 = mäßig, 3 = schwer). Unter Berücksichtigung der Ausdehnung des Ekzems (A, max. 5 P) wurde der Schweregrad (max. $60 \mathrm{P}$ ) nach folgender Formel berechnet:

$D A S I=(\mathbf{P} V+\mathbf{P E}+\mathbf{P} S+\mathbf{P I}) \times \mathbf{P A}$

Die Schweregradbeurteilung erfolgte vor Behandlungsbeginn (DASI 0), vor jeder UVA-Dosissteigerung (DASI 1, 2, 3 und 4) sowie nach Behandlungsende (DASI 5). Um das Kriterium der Beobachtungsgleichheit sicherzustellen, wurde die Bewertung stets von derselben Person durchgeführt. Die Patienten wurden bei jeder Therapiesitzung über evtl. aufgetretene unerwünschte Wirkungen befragt.

\section{Ergebnisse}

$\nabla$

Von fünfzehn eingeschlossenen Patienten gingen elf in die Endauswertung ein (8 Frauen, 3 Männer; mittleres Alter: 45,1 Jahre \pm 12,9; 28 - 66 Jahre). Ein Patient verließ auf eigenen Wunsch die Studie. Für drei Patienten wurde die Teilnahme an der Studie vorzeitig aus medizinischen Gründen beendet (bakterielle Sekundärinfektion nach erster Behandlung, akute Befundverschlechterung nach achter Behandlung, nicht studienbedingte Erkrankung). Der Erkrankungsbeginn lag im Mittel bei 39,7 Jahren $( \pm 14,5)$. Die aktuelle Dauer des Handekzems bei Behandlungsbeginn betrug 20 Monate $( \pm 29)$. Sieben Patienten beklagten ein chronisch rezidivierendes Auftreten, neun Patienten eine Therapieresistenz des Handekzems.

\section{Creme-PUVA-Behandlung}

Die Mediane der erhobenen DASI-Scores werden in $\triangle$ Abb. 1 grafisch dargestellt. Nach fünfzehn Behandlungen führte die Creme-PUVA-Behandlung zu einer signifikanten Reduktion des 


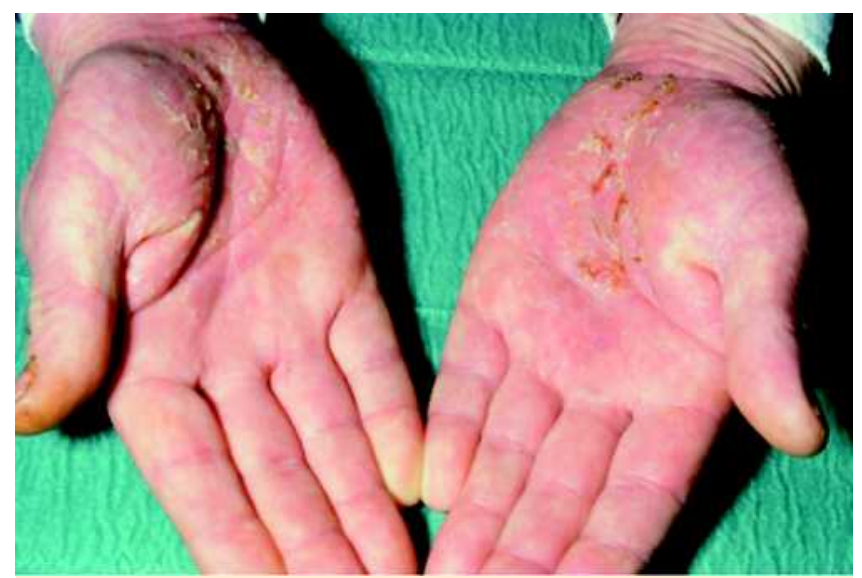

Abb. 2 Beispiel für einen Behandlungsverlauf. Patientenhände vor Behandlungsbeginn, rechte Hand DASI 36, linke Hand DASI 36, nach Randomisierung rechts UVA-1, links Creme-PUVA

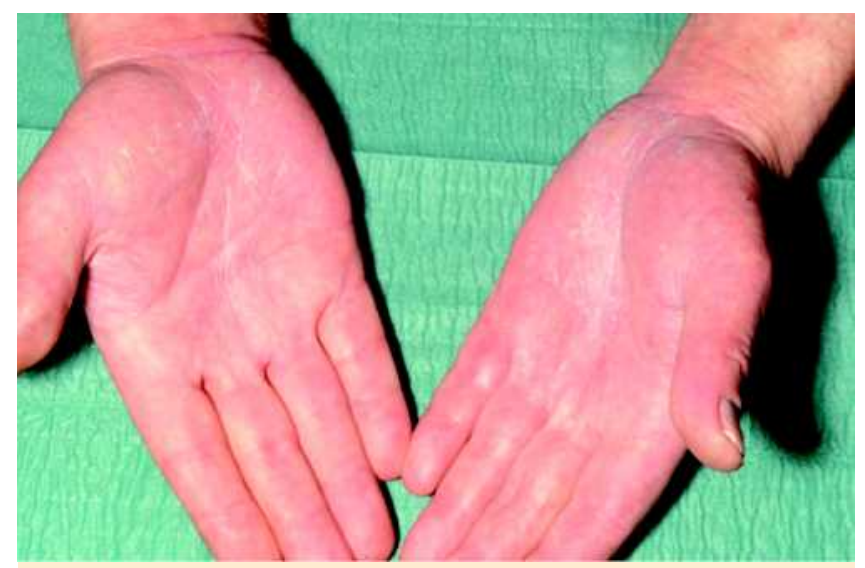

Abb. 3 Diese Patientenhände am Studienende, rechte Hand DASI 15 nach UVA-1, linke Hand DASI 24 nach Creme-PUVA.

DASI-Schweregradscores (Wilcoxon-Test für verbundene Stichproben: $\mathrm{p}=0,0498 ; \mathrm{n}=11$ ) um mehr als die Hälfte des Ausgangswertes.

\section{UVA-1-Therapie}

Die Mediane der erhobenen DASI-Scores werden in 1 Abb. 1 grafisch dargestellt. Nach fünfzehn Behandlungen führte die UVA-1-Behandlung zu einer signifikanten Reduktion des DASISchweregradscores (Wilcoxon-Test für verbundene Stichproben: p = 0,0039; $n=11$ ) um mehr als die Hälfte des Ausgangswertes.

\section{Vergleich beider Therapieformen}

In einem zweiten Schritt wurde die Wirksamkeit beider Therapieformen mit Hilfe des U-Tests von Mann und Whitney anhand der medianen DASI-Werte vor Behandlungsbeginn und nach Behandlungsende verglichen. Hier ergab sich ein p-Wert von $p=0,3070$. Damit konnte kein Unterschied zwischen den beiden Therapieformen bzgl. ihrer Wirksamkeit nachgewiesen werden. Der Vergleich beider Therapieformen wird in $\mathbf{A b b} .1$ grafisch dargestellt. Ein typischer Behandlungsverlauf ist in 1 Abb. 2 (Patientenhände vor Behandlungsbeginn; DASI re. Hand: $36 \mathrm{P}$; DASI li. Hand: $36 \mathrm{P}$ ) und $\bullet$ Abb. 3 (Patientenhände nach Behandlungsende; PUVA li. Hand: DASI 24 P; UVA-1 re. Hand: DASI 15 P) zu sehen.

\section{Nebenwirkungen}

Bei der Creme-PUVA-Therapie klagten fünf Patienten über eine gelegentliche Juckreizverstärkung im Anschluss an die Behandlung, drei berichteten über gelegentliches Brennen. Bei der UVA1-Therapie berichteten drei Patienten über eine gelegentliche Juckreizverstärkung, ein Patient über gelegentliches Brennen. Der Unterschied zwischen den beiden Behandlungsgruppen ist diesbezüglich nicht signifikant (Fisher's exakter Test, $\mathrm{p}=0,3426$ ). Gravierende Nebenwirkungen wie z.B. Blasenbildung traten bei keiner der beiden Therapien auf.

\section{Besprechung \\ $\nabla$}

Die Entwicklung der lokalen PUVA-Therapie ermöglichte es, einzelne umschriebene Hautbezirke wie Handflächen oder Fußsohlen zu therapieren, ohne die nicht betroffenen Hautareale wie bei der systemischen PUVA [10,17] unnötiger UV-Strahlung auszusetzen. Die sog. Creme-PUVA-Therapie ermöglicht eine gleichmäßige Verteilung des Photosensibilisators [20]. Ihre Wirksamkeit bei chronischen Hand- und Fußekzemen wurde bereits in mehreren Studien unter Beweis gestellt $[2,14,20]$.

Erste Erfolge mit dem sog. UVA-1-Licht, einem langwelligen Anteil des ultravioletten Lichts, konnten Krutmann et al. bei der Behandlung der akut exazerbierten atopischen Dermatitis erzielen [9]. In den darauffolgenden Jahren wurde diese Therapie u.a. bei der Morphea [4,6,7], dem systemischen Lupus erythematodes [16], der Urtikaria pigmentosa [12] und nicht zuletzt beim dyshidrosiformen Handekzem $[14,15,19]$ erfolgreich eingesetzt.

In der von uns durchgeführten Halbseitenstudie führte nach insgesamt fünfzehn Behandlungen sowohl die Creme-PUVA-Behandlung $(p=0,0498)$ als auch die UVA-1-Behandlung $(p=0,0039)$ zu einer signifikanten Reduktion des HandekzemSchweregrades, ein Unterschied zwischen beiden Therapieformen bzgl. ihrer Wirksamkeit konnte jedoch nicht nachgewiesen werden $(p=0,3070)$. Dies deckt sich mit den Ergebnissen in der von Petering et al. [14] 2004 veröffentlichten Studie. Bei gleicher Anzahl von Therapiesitzungen konnten wir annährend dieselben Ergebnisse, d.h. Reduktion des DASI um mehr als die Hälfte, mit einer höher dosierten 8-MOP-Creme $(0,001 \%$ in unserer Studie vs. 0,0006\%), aber mit wesentlich niedrigeren $\mathrm{ku}-$ mulativen Bestrahlungsdosen erzielen (UVA: 17,4 J/cm² vs. 130 $\mathrm{J} / \mathrm{cm}^{2}$; UVA-1: $600 \mathrm{~J} / \mathrm{cm}^{2}$ vs. $1720 \mathrm{~J} / \mathrm{cm}^{2}$ ).

Gravierende Nebenwirkungen traten bei keiner der beiden Therapieformen auf. Beklagt wurden lediglich eine vorübergehende Verstärkung des Juckreizes sowie gelegentliches Brennen. Der Unterschied zwischen beiden Behandlungsformen war diesbezüglich nicht signifikant ( $p=0,3426$ ). Es ist jedoch zu bedenken, dass bei der Creme-PUVA-Therapie ein potenzielles Langzeitrisiko für das Auftreten bestimmter Hautkrebsarten, einschließlich des Malignen Melanoms nicht ausgeschlossen werden kann. Dieses Risiko scheint mit der kumulativen UVA-Dosis bzw. der Anzahl der PUVA-Behandlungen zu steigen. Obwohl verläßliche Daten hierzu bislang nur für die orale PUVA-Therapie [11,21 - 23] existieren, sollte die Indikation auch für die topische PUVA-Therapie bei Risikopatienten zurückhaltend gestellt werden [25]. Mögliche Langzeitnebenwirkungen der UVA-1-Therapie sind derzeit noch nicht abzuschätzen. Mutzhas und Thomalla ermittelten in einem rechnerischen Verfahren für die UVA1-Therapie das geringste Langzeit-Nebenwirkungspozential bzgl. Hautalterung und Hautkrebsentwicklung verglichen mit anderen Phototherapieverfahren [13]. Da die Aktionsspektren 
vieler unerwünschter UV-bedingter Nebenwirkungen im kurzwelligen UV-Bereich liegen, kann spekuliert werden, dass die Nebenwirkungsrisiken einer UVA-1-Bestrahlung (340-400 nm) gering sind [8]. Mögliche Langzeitnebenwirkungen können derzeit dennoch nicht ausgeschlossen werden.

Bei der Therapie des dyshidrosiformen Handekzems sind sowohl die Creme-PUVA-Therapie als auch die UVA-1-Therapie wirksame Behandlungsmaßnahmen. Bei Betrachtung der einzelnen Patienten fallen jedoch individuelle Unterschiede im Ansprechen auf die eine oder andere Therapieform auf. Daher sollte für jeden Patienten die für ihn günstigste Behandlungsform durch Austesten der zur Verfügung stehenden Therapiealternativen und Berücksichtigung des Risiko- und Nebenwirkungsprofiles gefunden werden. Limitierend hierfür mag sein, dass UVA-1-Bestrahlungsgeräte, im Gegensatz zu PUVA-Teilbestrahlungsgeräten nur in wenigen Zentren verfügbar sind. Auch erschweren fehlende Daten bezüglich der Langzeitnebenwirkungen beider Therapieverfahren die Entscheidung. Umso wichtiger ist es, die niedrigste, aber dennoch effektive Bestrahlungsdosis zu evaluieren. Beide Therapien haben in den verwendeten niedrigen Dosierungen in dieser Studie ihre Wirksamkeit unter Beweis gestellt.

\section{Abstract}

\section{Medium-Dose-UVA-1 Irradiation - and Topical PUVA - Therapy in Chronic Dyshidrotic Hand Dermatitis - A Prospective Randomized Study \\ $\nabla$}

This study has aimed to compare two different therapy schedules for dishydrotic hand eczema, medium-dose UVA-1- irradiation and topical PUVA-treatment (topical psoralen and UVA). 15 patients were included in a prospective randomised study (right versus left hand) for five weeks with irradiations $3 \times$ per week. The hand treated with $0.001 \%$ 8-Methoxypsoralen-cream (topical PUVA) was irradiated with weekly increasing UVA-doses (cumulative UVA-dose $17,4 \mathrm{~J} / \mathrm{cm}^{2}$ ). Medium-dose-UVA-1 irradiation was maintained at $40 \mathrm{~J} / \mathrm{cm}^{2}$ (cumulative UVA-1-dose $600 \mathrm{~J} / \mathrm{cm}^{2}$ ). To compare therapeutical effects, a grading with DASI (Dyshidrotic Eczema Area and Severity Index) was performed. Out of 15 patients included in the study, it was possible to evaluate 11 at the end $(1 \times$ non compliance, $1 \times$ bacterial infection, $1 \times$ exacerbation, $1 \times$ disease not related to the study). 15 irradiation sessions with topical-PUVA-therapy $(\mathrm{p}=0.0498 ; \mathrm{n}=11)$, as well as UVA-1-therapy ( $\mathrm{p}=0.0039 ; \mathrm{n}=11$ ) resulted in a significant decrease of the DASI compared to study start (Wilcoxon-test). With a p-value of $p=0.3070$ it was not possible to identify any differences between the two therapy schedules in respect of efficacy (U-test of Mann and Whitney). Each treatment schedule was effective (reduction of DASI to more than half of the value) and safe. Therapeutic efficacy was shown with lower cumulative doses than published hitherto.

\section{Literatur}

1 Bayerl C, Garbea A, Peiler D, Rzany B, Allgäuer T, Kleesz P, Jung EG, Frosch PJ. Pilotstudie zur Therapie des beruflich bedingten Handekzems mit einer neuen tragbaren UVB-Bestrahlungseinheit. Akt Dermatol 1999; 25: $302-305$

2 Grundmann-Kollmann M, Behrens S, Peter RU, Kerscher M. Treatment of severe recalcitrant dermatoses of the palms and soles with PUVA- bath versus PUVA-cream therapy. Photodermatol Photoimmunol Photomed 1999; 15: 87-89

3 Grundmann-Kollmann M, Tegeder I, Ochsendorf FR, Zollner TM, Ludwig $R$, Kaufmann R, Podda M. Kinetics and dose-response of photosensitivity in cream psoralen plus ultraviolet A photochemotherapy: comparative in vivo studies after topical application of three standard preparations. Br J Dermatol 2001; 144: 991 - 995

4 Gruss CJ, von Kobyletzki G, Behrens-Williams SC, Lininger J, Reuther T, Kerscher M, Altmeyer P. Effects of low dose ultraviolet A-1 phototherapy on morphea. Photodermatol Photoimmunol Photomed 2001; 17: $149-155$

5 Halpern SM, Anstey AV, Dawe RS, Diffey BL, Farr PM, Ferguson J, Hawk JLM, Ibbotson S, McGregor JM, Murphy GM, Thomas SE, Rhodes LE. Guidelines for topical PUVA: a report of a workshop of the British Photodermatology Group. Br J Dermatol 2000; 142: 22 - 31

6 Kerscher M, Dirschka T, Volkenandt M. Treatment of localised scleroderma by UVA-1 phototherapy. Lancet 1995; 346: 1166

7 Kerscher M, Volkenandt M, Gruss C, Reuther T, von Kobyletzki G, Freitag $M$, Dirschka T, Altmeyer P. Low-dose UVA phototherapy for treatment of localized scleroderma. J Am Acad Dermatol 1998; 38: 21 - 26

8 Kowalzick L, Mensing H, Wagner G. Grundlagen. In: Kowalzick L, Mensing $\mathrm{H}$, Wagner G (Hrsg). Praxis der Lichtdermatosen - Diagnostik, Therapie und Prävention. Bremen: UNI-MED, 2000: 15-20

9 Krutmann J, Czech W, Diepgen T, Niedner R, Kapp A, Schöpf E. High-dose UVA 1 therapy in the treatment of patients with atopic dermatitis. J Am Acad Dermatol 1992; 26: 225 - 230

10 LeVine MJ, Parrish JA, Fitzpatrick TB. Oral Methoxsalen Photochemotherapy (PUVA) of Dyshidrotic Eczema. Acta Derm Venereol 1981; 61: $570-571$

11 Lindelöf B, Sigurgeirsson B, Tegner E, Larkö O, Johannesson A, Berne B, Christensen OB, Andersson T, Törngren M, Molin L, Nylander-Lundqvist E, Emtestam L. PUVA and cancer: a large-scale epidemiological study. Lancet 1991; 338: $91-93$

12 Meffert $H$. Stellenwert der UVA-1-Therapie; Tagungsbericht der 109. Tagung der Berliner Dermatologischen Gesellschaft am 2. März 1996. Z Hautkr 1996; 71: 721 - 738

13 Mutzhas MF, Thomalla E. Photobiologisch bewertete UV-Nebenwirkungs-Risiken bei fünf verschiedenen Phototherapieverfahren zur Behandlung der Neurodermitis. Akt Dermatol 1994; 20: 35-40

14 Petering H, Breuer C, Herbst R, Kapp A, Werfel T. Comparison of localized high-dose UVA1 irradiation versus topical cream psoralen-UVA for treatment of chronic vesicular dyshidrotic eczema. J Am Acad Dermatol 2004; 50: 68 - 72

15 Polderman MCA, Govaert JCM, Le Cessie S, Pavel S. A double-blind placebo-controlled trial of UVA- 1 in the treatment of dyshidrotic eczema. Clin Exp Dermatol 2003; 28: 584-587

16 Polderman MCA, Huizinga TWJ, Le Cessie S, Pavel S. UVA-1 cold light treatment of SLE: a double blind, placebo controlled crossover trial. Ann Rheum Dis 2001; 60: 112 - 115

17 Rosén K, Mobacken H, Swanbeck G. Chronic Eczematous Dermatitis of the Hands: A Comparison of PUVA and UVB Treatment. Acta Derm Venereol 1987; 67: 48 - 54

18 Schempp CM, Müller H, Czech W, Schöpf E, Simon JC. Treatment of chronic palmoplantar eczema with local bath-PUVA therapy. J Am Acad Dermatol 1997; 36: $733-737$

19 Schmidt T, Abeck D, Boeck K, Mempel M, Ring J. UVA-1 Irradiation is Effective in Treatment of Chronic Vesicular Dyshidrotic Hand Eczema. Acta Derm Venereol 1998; 78(4): 318-319

20 Stege H, Berneburg M, Ruzicka T, Krutmann J. Creme-PUVA-Photochemotherapie. Hautarzt 1997; 48: 89-93

21 Stern RS, Laird N. The Carcinogenic Risk of Treatments for Severe Psoriasis. Cancer 1994; 73: 2759-2764

22 Stern RS, Nichols KT, Väkevä LH. Malignant melanoma in patients treated for psoriasis with methoxsalen (psoralen) and ultraviolet A radiation (PUVA). N Engl J Med 1997; 336: 1041 - 1045

23 Stern RS, Thibodeau LA, Kleinermann RA, Parrish JA, Fitzpatrick TB. Risk of cutaneous carcinoma in patients treated with oral methoxsalen photochemotherapy for psoriasis. N Engl J Med 1979; 300: 809-813

24 Vocks A, Plötz SG, Ring J. The Dyshidrotic Eczema Area and Severity Index - A Score Developed for the Assessment of Dyshidrotic Eczema. Dermatology 1999; 198: 265-269

25 Wolff K. Photochemotherapie (PUVA): Pro und Contra. Hautarzt 1985; 36: $25-33$ 